\title{
Molecular Profiling and Novel Therapeutic Strategies for Mucosal Melanoma: A Comprehensive Review
}

\author{
Alice Indini ${ }^{1}(\mathbb{D})$, Fausto Roila ${ }^{2}$, Francesco Grossi ${ }^{1,3}\left(\mathbb{D}\right.$, Daniela Massi ${ }^{4}$ and Mario Mandalà ${ }^{2, *(D)}$ \\ 1 Division of Medical Oncology, Department of Medicine and Surgery, Ospedale di Circolo e Fondazione \\ Macchi, ASST dei Sette Laghi, 21100 Varese, Italy; alice.indini@gmail.com (A.I.); \\ francesco.grossi@asst-settelaghi.it (F.G.) \\ 2 Unit of Medical Oncology, Department of Surgery and Medicine, University of Perugia, 06129 Perugia, Italy; \\ fausto.roila@unipg.it \\ 3 Faculty of Medicine and Surgery, University of Insubria, Ospedale di Circolo e Fondazione Macchi, \\ 21100 Varese, Italy \\ 4 Section of Pathological Anatomy, Department of Health Sciences, University of Florence, 50134 Florence, Italy; \\ daniela.massi@unifi.it \\ * Correspondence: mario.mandala@unipg.it
}

check for updates

Citation: Indini, A.; Roila, F.; Grossi, F.; Massi, D.; Mandalà, M. Molecular Profiling and Novel Therapeutic Strategies for Mucosal Melanoma: A Comprehensive Review. Int. J. Mol. Sci. 2022, 23, 147. https:// doi.org/10.3390/ijms23010147

Academic Editor: Silvana Morello

Received: 29 November 2021

Accepted: 22 December 2021

Published: 23 December 2021

Publisher's Note: MDPI stays neutral with regard to jurisdictional claims in published maps and institutional affiliations.

Copyright: (C) 2021 by the authors. Licensee MDPI, Basel, Switzerland. This article is an open access article distributed under the terms and conditions of the Creative Commons Attribution (CC BY) license (https:// creativecommons.org/licenses/by/ $4.0 /)$.

\begin{abstract}
Mucosal melanoma is a rare and aggressive subtype of melanoma. Unlike its cutaneous counterpart, mucosal melanoma has only gained limited benefit from novel treatment approaches due to the lack of actionable driver mutations and poor response to immunotherapy. Over the last years, whole-genome and exome sequencing techniques have led to increased knowledge on the molecular landscape of mucosal melanoma. Molecular studies have underlined noteworthy findings with potential therapeutic implications, including the presence of KIT mutations, which are potential targets of tyrosine kinase inhibitors currently in use in the clinic (imatinib), but also SF3B1 mutation, $C D K 4$ amplifications, and $C D K N 2 A$ gene deletions, which are presently under investigation in clinical trials. Recent results from a pooled analysis of patients with mucosal melanoma treated with immunotherapy have suggested that the combination of immune checkpoint inhibitors might improve survival outcomes in this subset of patients, as compared with single-agent immunotherapy. However, these results are not confirmed across different studies, and combo-immunotherapy correlates with a higher rate of adverse events. In this review, we describe the clinical, biological, and genetic features of mucosal melanoma. We also provide an update on the results of approved systemic treatment in this setting and overview the therapeutic strategies currently under investigation in clinical trials.
\end{abstract}

Keywords: melanoma; mucosal; immunotherapy; targeted therapy; c-kit

\section{Introduction}

Mucosal melanoma is a rare disease that is epidemiologically, biologically, and molecularly distinct from cutaneous melanoma [1]. Non-cutaneous melanocytes can be found in multiple sites of the human body, namely the ocular tract (including the uvea and the conjunctiva) and mucosal surfaces lining respiratory, gastrointestinal, and urogenital tracts [2]. With an overall incidence of 1.4 cases/million, mucosal melanomas represent approximately $1.5 \%$ of all melanoma cases and only $0.03 \%$ of all diagnosed cancers [3]. Mucosal melanomas most often arise in the head and neck cavities (55.4\%), including the oral and nasal cavity and accessory sinuses, followed by the anus/rectum $(23.8 \%)$ and the female genital tract (18\%) (Figure 1) [3,4]. A variety of primary non-cutaneous melanoma has been described to occur in almost every part of the body, including the gastrointestinal tract, larynx, lungs, leptomeninges, or biliary tree [5,6]. However, in some cases, melanomas arising in atypical internal sites might represent metastases of occult primary cutaneous melanoma, as suggested by mutational analysis of a cohort of patients with presumed 
primary pulmonary melanomas, showing a high mutational load with ultraviolet (UV) signature and mutations in genetic drivers typical of cutaneous melanoma [7].
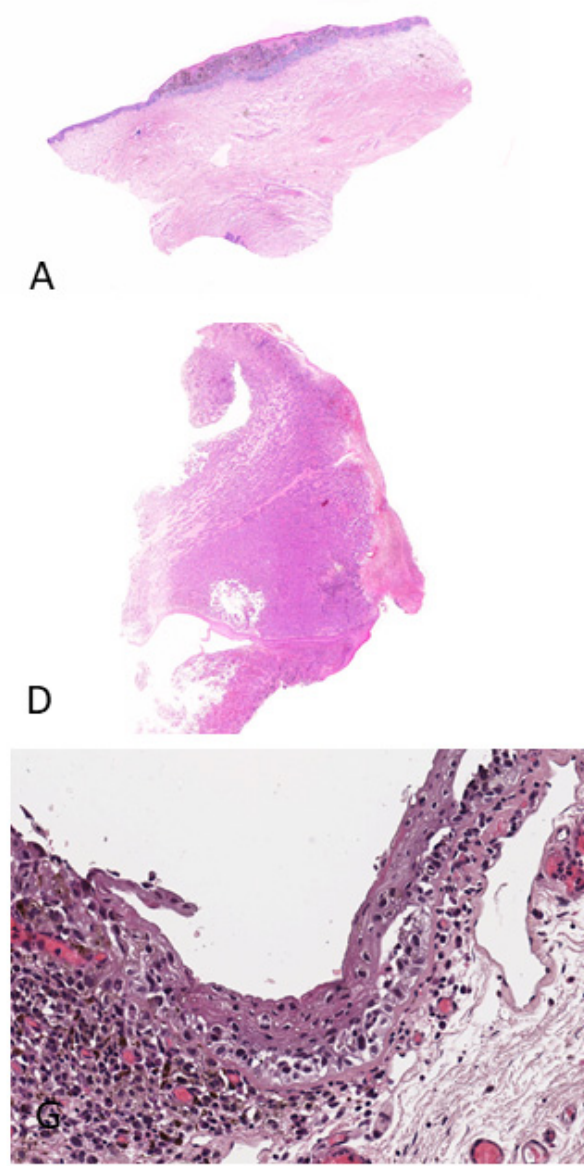
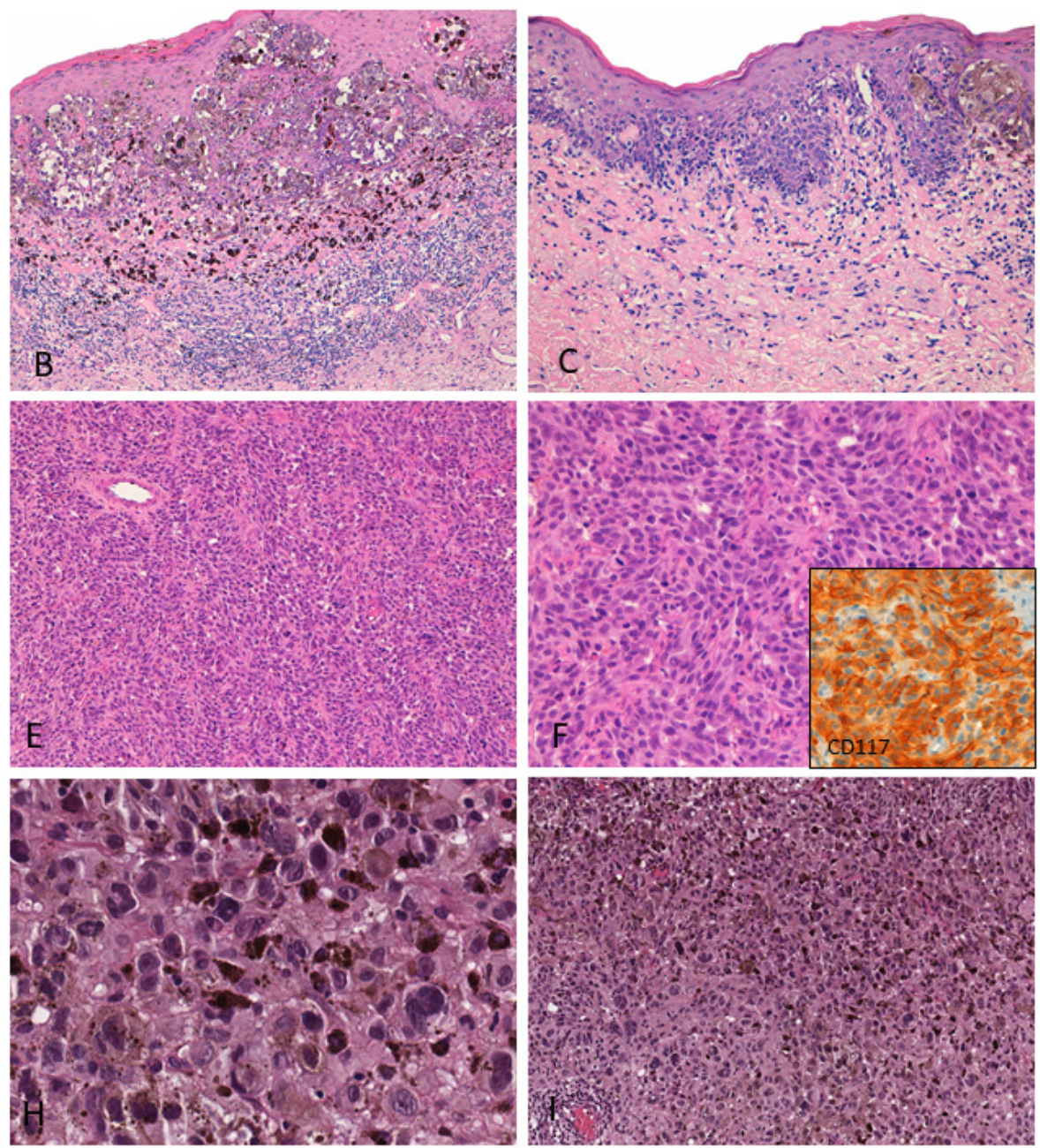

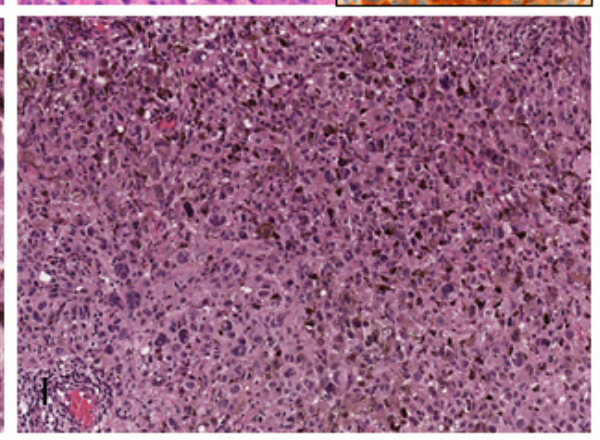

Figure 1. (A-C) Female, 76 years-Mucosal melanoma on the vulva (labia minora) characterized by atypical pigmented melanocytes in single units and nests with pagetoid spread; a brisk lymphocytic infiltrate with numerous melanophages is observed in the upper dermis; (D-F) Male, 86 yearsEndoscopic biopsy reveals a bulky tumor diagnosed as amelanotic mucosal melanoma of the anal canal; the tumor shows diffuse proliferation of non-pigmented epithelioid cells; tumor cells show cytoplasmic and membranous CD117 staining (inset); (G-I) Female, 47 years-Highly pigmented conjunctival mucosal melanoma (left eye) with prominent melanin pigment in intracellular and extracellular location; both in situ $(\mathbf{G})$ and invasive components $(\mathbf{H}, \mathbf{I})$ are shown. These images come from the pathology archive of patients with mucosal melanomas, as indicated in the "Materials and methods" section.

Compared to its cutaneous counterpart, mucosal melanoma represents a challenge for both diagnosis and treatment. Due to their hidden sites of origin, these tumors are often asymptomatic in their early stages and are usually diagnosed late. The presence of a thick vascularization on the site of disease onset allows early metastatic spread through the lymphatic and vascular networks. Altogether, these features explain the aggressive biology of mucosal melanomas, their overall poor prognosis, and low survival rates [1]. Moreover, the rarity of the disease makes large epidemiological studies and clinical trials difficult to be performed. Notwithstanding, over the last few years, the therapeutic perspective has started to change thanks to the growing knowledge on the molecular pathogenesis of 
mucosal melanoma and the use of novel treatment strategies. Still, most evidence suggests that the prognosis of patients with mucosal melanoma has not improved as much as for cutaneous melanoma [8]. In this review, we describe the clinical and biological features of mucosal melanoma, focusing on a small case presentation from our clinical experience. We also provide an update on the results of approved systemic treatment in this setting and overview the therapeutic strategies currently under investigation in clinical trials.

\section{Materials and Methods}

The papers referenced in this review were selected through a PubMed search performed on 1 November 2021, with the following searching terms: melanoma and mucosal, or non-cutaneous, or head and neck, genitourinary, or anal, or vulvovaginal. Oral presentation, abstracts, and posters presented at the American Society of Clinical Oncology (ASCO, Alexandria, VA, USA) 2021 and the European Society for Medical Oncology (ESMO, Lugano, Switzerland) 2021 annual meetings were retrieved for data on mucosal melanoma. We also overview ongoing research and data of combination therapies currently under investigation, which will impact future therapeutic strategies. Clinicaltrials.gov was searched to identify ongoing clinical trials enrolling patients with mucosal melanoma, both in the adjuvant and systemic settings.

Histologic and radiologic documentation were retrieved from patients' clinical records in order to provide representative data upon patients' (or their relatives') written informed consent to use these images for research publication purposes (Figures 1 and 2).

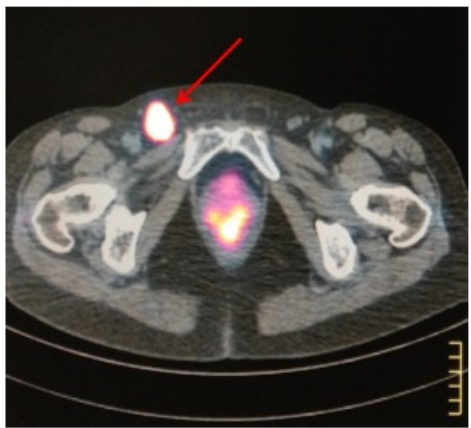

Panel A: baseline PET/CT scan

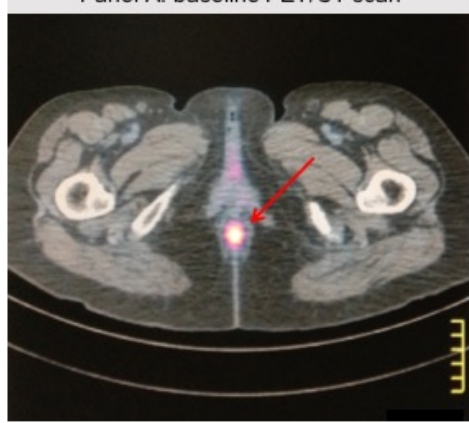

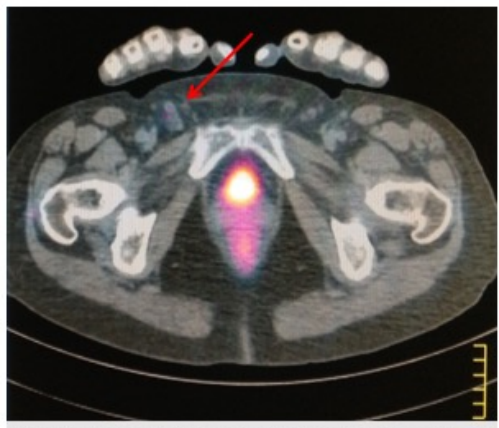

Panel B: PET/CT scan after Imatinib treatment

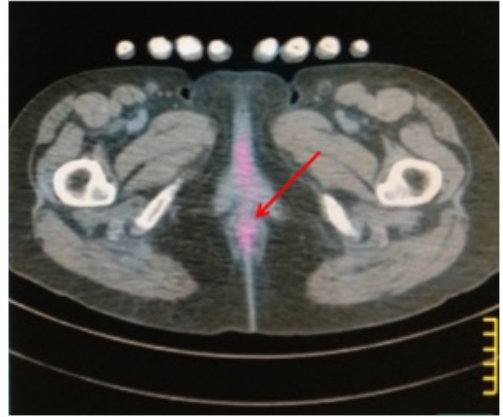

Figure 2. A 67-year-old patient came to our attention because of rectorrhagia; a PET/CT scan showed lung and abdominal lymph node metastases. A biopsy of the anal mass showed atypical epithelioid cells with pleomorphic features. Immunohistochemistry showed strong positivity for HMB45 and Melan A, whereas cytokeratins and S-100 expression were negative, consistent with the diagnosis of anal mucosal melanoma. c-KIT staining was positive in $>75 \%$ of tumor cells, and DNA sequence analysis revealed a KIT-activating mutation (L576P) in exon 11. The patient received Imatinib $400 \mathrm{mg} /$ die. Panel (A) shows the anal baseline lesion with a pathologic left inguinal lymph node. Panel (B) shows the PET/CT scan after 4 months of Imatinib treatment, showing a near-complete response in the anal lesion and the inguinal lymph node. The response lasted 3 years, then the patient progressed and died. These images come from the radiology archive of a patient with mucosal melanomas, as indicated in the "Materials and methods" section. 


\section{Genomic Profiling}

Genetic alterations occurring in mucosal melanoma are different from those observed in cutaneous melanoma. Recently, a meta-analysis of major studies of whole genome and whole exome sequencing of mucosal melanoma has been published [9]. Compared to cutaneous forms, mucosal melanomas show lower rates of somatic mutations, do not display the UV mutational signatures and show increased somatic genomic instability [9]. The overall frequency of main mutations in mucosal melanomas is as follows (Table 1): NRAS (8\%), BRAF (6\%), neurofibromin 1 (NF1) (14\%), KIT (13\%), splicing factor $3 b$ subunit 1 (SF3B1) (15\%) [9]. Other less commonly described mutations in mucosal melanomas are TP53 (8.9\%), sprout-related EVH1 domain containing protein 1 (SPRED1) $(7 \%)$, ATP dependent helicase (ATRX) $(6 \%)$, and Chromodomain Helicase DNA Binding Protein 8 (CHD8) (4\%) [10-12]. However, the frequency of different mutations may vary across different studies and reports. Moreover, there are significant differences in the frequency of these mutations across specific anatomical sites of mucosal melanomas [9]. Head and neck melanomas, and specifically sinonasal melanomas, most commonly show mutations in NRAS and Telomerase Reverse Transcriptase (TERT) promoter, as compared with $B R A F$ and KIT mutations [13-15]. Genitourinary tract and anorectal melanomas (both in males and females) frequently show KIT mutations and amplifications, together with NRAS mutations; notably, KIT mutations are uncommon in vaginal melanomas but are frequently present in vulvar melanomas [16]. Recurrent loss of SPRED1, a negative regulator of the mitogen-associated protein kinase (MAPK) pathway which acts by recruiting NF1 to the plasmatic membrane, has been found in $37 \%$ of 47 patients with mucosal melanomas, mostly of anorectal and vulvovaginal origin: this molecular alteration was mutually exclusive with NF1 mutations, which were found in $12 \%$ of patients [17]. In a study utilizing a zebrafish model, Ablain et al. demonstrated an in vivo cooperation between loss of SPRED1 and KIT activating mutations, suggesting that this molecular alteration might induce cell proliferation and confer resistance to KIT inhibitors [17].

Mutations in BRAF and NRAS are not so uncommon among mucosal melanomas (up to $20 \%$ of cases); rather, they have peculiar features that are radically different from those observed in cutaneous melanomas [18]. Mucosal melanomas show a lower frequency of NRAS Q61 mutations and a higher frequency of mutations in G12 and G13, suggesting that these are not linked to UV irradiation but possibly due to the effect of external genotoxic factors, which remain to be identified. Similarly, BRAF mutations in mucosal melanomas mainly consist of non-canonical non-V600 mutations and are similar to those observed in lung adenocarcinomas for both the location of mutated codons and the preferred amino acid substitutions (mostly prevalence of D594G, G469A, and K601E) [18]. Indeed, BRAF mutation was reported in $26 \%$ of patients with vulvar and vaginal melanoma in a case series of 51 patients; however, only $50 \%$ of them were V600 mutations [16]. BRAF fusions in mucosal melanomas have also been described, with a comparable frequency as of cutaneous triple wild type melanoma (i.e., lacking $B R A F, N R A S$, and NF1 mutations) [19]. Interestingly, while being resistant to vemurafenib in vitro, the ZNF767-BRAF fusion showed in vitro and in vivo response to MEK inhibitor alone or combined with either Phosphatidylinositol4,5-Bisphosphate 3-Kinase (PI3K) or cyclin-dependent kinases (CDK) 4/6 inhibitors [19]. The distribution of $B R A F$ and NRAS mutation varies according to geographic regions, as observed in cutaneous melanoma, and might explain the better survival observed among European patients in comparison with patients from North America and Asia [20].

Loss of NF1, which acts as a negative regulator of Ras, is associated with increased MAPK pathway activity [21]. The rate of NF1 mutations is comparable across cutaneous and mucosal melanomas [9]. Interestingly, the whole-exome sequencing study by Hintzsche et al. found that NF1 was co-mutated with KIT in 32\% of mucosal melanomas, which is a significantly higher rate if compared with cutaneous forms [22].

SF3B1 is a spliceosomal protein that plays a major role in RNA splicing. Thus, SF3B1 mutations result in alternative splicing leading to an overall transcriptomic dysregulation [23]. SF3B1 mutations, specifically the $\mathrm{R} 625 \mathrm{C}$ and $\mathrm{R} 625 \mathrm{H}$, are the most commonly 
reported in uveal, vulvovaginal, and anorectal melanomas [22,24,25]. SF3B1 mutation has been reported to be associated with late metastatization and better prognosis in patients with uveal melanoma [25]; however, it did not seem to have the same correlation in a recently published case series of mucosal melanomas [19]. Comparison of mutational profiles of upper versus lower body sites mucosal melanomas found SF3B1 hotspot mutations in $27 \%$ of lower body (most commonly vulvar ad anorectal primary melanomas) compared to $6 \%$ in the upper body sites [9]. Conversely, nasal melanomas more frequently showed non-canonical SF3B1-E1105B mutations located in the heat domain of SF3B1 [22].

Mutations in Insulin Like Growth Factor Receptor 2 (IGF2R) and Deleted in Colorectal Cancer (DCC) genes were reported to be strikingly more frequent among mucosal as compared with cutaneous melanomas, accounting for nearly $32 \%$ of patients in the study by Iida et al. According to the Tumor Cancer Genome Atlas (TCGA) database, such mutations are quite uncommon in other types of solid tumors, thus confirming the peculiar and unique genetic background of mucosal melanomas [12].

Anaplastic lymphoma kinase $(A L K)$ fusions have been described in mucosal melanoma, specifically the EML4-ALK fusion and an alternate $A L K$ isoform, $A L K-A T 1$. However, such molecular alterations have no clear in vitro and in vivo sensitivity to ALK inhibition, and the therapeutic impact is yet to be clarified [26].

Table 1. Summary of most common genetic alterations in mucosal melanoma $[9,13-19,22-25,27,28]$.

\begin{tabular}{|c|c|c|c|c|}
\hline Gene & Molecular Alteration (s) & Frequency & Distribution across Melanoma Subtypes & Therapeutic Implications \\
\hline \multirow{3}{*}{ BRAF } & V600 & $6 \%$ & $\begin{array}{l}\text { similar rates in upper and lower } \\
\text { body regions }\end{array}$ & $\begin{array}{l}\text { MAPK inhibition (BRAF and } \\
\text { MEK inhibitors) }\end{array}$ \\
\hline & $\begin{array}{c}\text { D594G } \\
\text { G469A, G469A } \\
\text { K601E } \\
\text { L505H, L597R } \\
\text { T599I }\end{array}$ & $5-20 \%$ & \multirow[t]{2}{*}{ N.A. } & $\begin{array}{l}\text { Unknown response to } \\
\text { MAPK inhibition }\end{array}$ \\
\hline & ZNF767 fusion & N.A. & & $\begin{array}{l}\text { MEK inhibitor }+/- \text { PI3K or } \\
\text { CDK } 4 / 6 \text { inhibitors }\end{array}$ \\
\hline NRAS & $\begin{array}{l}\text { Q61 } \\
\text { G12 } \\
\text { G13 }\end{array}$ & $8-10 \%$ & $\begin{array}{l}43 \% \text { vaginal melanomas } \\
37.5 \% \text { esophageal melanomas }\end{array}$ & Potential role of MEK inhibitors \\
\hline KIT & $\begin{array}{l}\text { Amplifications and missense } \\
\text { mutations (Ex11 and Ex12-21) }\end{array}$ & $13 \%$ & $\begin{array}{l}\text { similar rates in upper and lower } \\
\text { body regions }\end{array}$ & $\begin{array}{l}\text { Imatinib } \\
\text { Dasatinib } \\
\text { Nilotinib }\end{array}$ \\
\hline NF1 & Loss of function mutations & $14 \%$ & $\begin{array}{l}10 \% \text { upper body regions } \\
18 \% \text { lower body regions }\end{array}$ & Potential role of MEK inhibitors \\
\hline SF3B1 & R625 & $15 \%$ & $\begin{array}{l}6 \% \text { upper body regions } \\
27 \% \text { lower body regions }\end{array}$ & H3B-8800 (clinical trials) \\
\hline SPRED1 & Loss of function mutations & $37 \%$ & $\begin{array}{l}\text { most common among anorectal and } \\
\text { vulvovaginal melanomas }\end{array}$ & $\begin{array}{l}\text { No actual clinical applications } \\
\text { Potential resistance to } \\
\text { KIT inhibitors }\end{array}$ \\
\hline $\begin{array}{l}\text { TERT } \\
\text { CDK4 }\end{array}$ & Co-amplification & N.A. & $>50 \%$ oral melanomas & $\begin{array}{l}\text { Potential role of } \\
\text { CDK4/6 inhibitors }\end{array}$ \\
\hline
\end{tabular}

Abbreviations: CDK4/6: cyclin-dependent kinase 4/6; MAPK: mitogen-associated protein kinase; N.A.: not assessed; PI3K: Phosphatidylinositol-4,5-Bisphosphate 3-Kinase.

Recent studies of mucosal melanoma analyzed by next-generation sequencing demonstrated that mucosal melanomas have high chromosomal instability and often show structural variants of CDK4, MDM2, TERT, cyclin D1 (CCND1), and NOTCH2, leading to their amplification, along with losses of cyclin-dependent kinase inhibitor (CDKN) 2A/B, phosphatase and tensin homolog (PTEN) and p53 [10,11,17]. Several tumor-intrinsic factors leading to immune escape and resistance to immune-checkpoint inhibitors have been characterized in cutaneous melanoma, including low tumor antigenicity, disruption of interferongamma (IFN- $\gamma$ ) signature, and loss of major histocompatibility complex (MHC) expression. 
Moreover, PTEN loss, amplification of MAPK and CDK4/ 6 pathways, and WNT- $\beta$ catenin dysregulation are all oncogenic signals contributing to immunosuppression [27]. However, the role of such mechanisms in mucosal melanomas is still unknown [28]. Recurrent activating mutations in $\beta$-catenin gene (CTNNB1) were detected in mucosal melanomas of various primary sites, suggesting they might play a role in resistance to immunotherapy [10]. Moreover, the lower antigenic load, low tumor mutational burden, and programmed cell death ligand 1 (PD-L1) expression of non-cutaneous melanomas together with a higher level of aneuploidy might be associated with lower $\mathrm{T}$ cell activation and poor response to immunotherapy $[12,29]$.

In conclusion, mucosal melanomas show a complex and peculiar genomic profile that contributes to their biology, aggressiveness, and accounts for the poor response to systemic therapies. Epigenetic mechanisms further complicate this picture, as they might influence response to treatment [30]. Several therapeutic strategies, mostly leveraged from previous experience of cutaneous melanoma studies, have been investigated in mucosal melanoma and will be detailed in the next section.

\section{Therapeutic Approach}

\subsection{Targeted Therapy and Tyrosine Kinase Inhibitors}

Given the low rate of BRAF V600 mutation among patients with mucosal melanomas, the combination of BRAF and MEK inhibitors has not led to the same clinical success observed in cutaneous melanoma harboring BRAF activating mutations. Still, there is preclinical evidence that NF1 loss of function mutations or deletions might lead to increased resistance to BRAF inhibitors [31]. As such, NF1 mutations together with RAS mutations and $B R A F$ fusions, which have been described in mucosal melanomas and lead to increased activation of the MAPK pathway, are promising therapeutic targets for MEK inhibition.

Certain KIT alterations, namely the KIT exon 11 and 13 mutations, show response to KIT inhibitors such as imatinib, nilotinib, and dasatinib [32,33]. Figure 2 shows PET/CT scan images of a patient with KIT exon 11 mutated anorectal mucosal melanoma responding to first-line treatment with imatinib. Conversely, KIT exon 17 mutation, KIT amplification, or immunohistochemistry staining positivity on tumor tissue appear to have minimal or no sensitivity to KIT inhibitors. A recent meta-analysis of 19 studies of c-Kit inhibitors for unresectable or metastatic mucosal, acral, or chronically sun-damaged melanoma (overall sample size of 601 patients) showed a pooled objective response rate (ORR) of $14 \%$ for all inhibitors among patients with mucosal melanoma, with the highest ORR (20\%) for nilotinib [34]. Serial tumor biopsies of patients treated with nilotinib in a French phase II clinical trial showed that patients with good response to nilotinib had persistently decreased levels of phospho-STAT3 as compared with poor responders to treatment [35]. This data underlines the potential role of phospho-STAT3 as a biomarker of response to nilotinib through increased activity of KIT inhibition through the downregulation of the downstream signaling protein STAT3.

The dysregulation of cell cycle progression, caused by CDK4 amplification and/or CCND1 amplification and/or p16 (CDKN2A) loss, is a key genetic feature in mucosal melanoma [11]. Targeting CDK4/6 is a promising therapeutic strategy that is supported by a strong preclinical rationale and some case reports of response to the CDK4/ 6 inhibitor, palbociclib [11,36,37]. To date, the use of CDK4/6 inhibitors in patients with mucosal melanoma harboring amplification of CDK4 is under evaluation in clinical trials (Table 2).

SF3B1 mutation represents another promising target among patients with mucosal melanoma. Currently, the orally available spliceosomal inhibitor, H3B-8800, is under investigation in phase 1 clinical trials of patients with advanced myeloid malignancies harboring SF3B1-mutations [38]. Another therapeutic strategy that demonstrated antitumor activity in Asian patients with acral and mucosal melanoma after the failure of conventional treatment was the combination of an alkylating agent, temozolomide, with the vascular endothelial growth factor receptor 2 (VEGFR2) tyrosine kinase inhibitor, apatinib [39]. 
However, due to the limited efficacy observed in a single phase 1 study, this combination was not developed further.

\subsection{Immunotherapy}

The role of immune-checkpoint inhibitors (ICIs), including antibodies targeting the cytotoxic T lymphocyte 4 (CTLA-4), ipilimumab, and the programmed cell death 1 (PD-1), nivolumab, and pembrolizumab, as monotherapies in mucosal melanoma, has been assessed in several monocentric and multicentric retrospective series [40-43]. Overall, ORR and progression-free survival (PFS) rates have been found to be comparable, but most often worse, compared with cutaneous melanomas, regardless of the primary site of origin of mucosal melanomas. A post-hoc analysis of three trials using pembrolizumab in metastatic melanoma, the KEYNOTE-001, KEYNOTE-002, and KEYNOTE-006, showed an ORR of $19 \%$ (95\% CI, 11-29\%), median PFS of 2.8 months (95\% CI, 2.7-2.8), and the median OS was 11.3 months (95\% CI, 7.7-16.6) among 84 patients with mucosal melanoma out of 1567 total patients enrolled [44]. Nivolumab monotherapy in patients with rare melanoma subtypes who progressed on or after ipilimumab treatment was evaluated in the phase II CheckMate 172 study [45]. This trial enrolled 1008 patients, of whom $6.3 \%(n=63)$ had mucosal melanoma. Results from this trial showed that both mucosal and ocular melanomas had lower median overall survival (OS) compared with acral cutaneous and non-cutaneous melanoma, being median OS 11.5 months (95\% CI, 6.4-15.0) and 18 month OS rates 31.5\% for patients with mucosal melanoma [45].

In a pooled analysis of studies using nivolumab monotherapy, including 86 patients with mucosal melanoma, median PFS was 3.0 months (95\% CI, 2.2 to 5.4 months) and 6.2 months (95\% CI, 5.1 to 7.5 months) for mucosal and cutaneous melanoma, with objective response rates of $23.3 \%$ (95\% CI, $14.8 \%$ to $33.6 \%$ ) and $40.9 \%$ (95\% CI, $37.1 \%$ to $44.7 \%)$, respectively [46]. The combination of nivolumab and ipilimumab has yielded more promising results even among patients with mucosal melanomas, with improved outcomes compared with both agents used as monotherapies [47,48]. In the pooled analysis of nivolumab studies, median PFS in patients treated with combined nivolumab and ipilimumab was 5.9 months (95\% CI, 2.8 months to not reached) and 11.7 months (95\% CI, 8.9 to 16.7 months) for mucosal and cutaneous melanoma, and the ORR was $37.1 \%$ (95\% CI, $21.5 \%$ to $55.1 \%$ ) and $60.4 \%(95 \% \mathrm{CI}, 54.9 \%$ to $65.8 \%)$, respectively [46]. The 5-year follow-up of 79 patients with mucosal melanoma from the CheckMate-067 trial showed that the combination of ipilimumab and nivolumab resulted in considerable higher ORR compared with ipilimumab alone ( $43 \%$ vs. $7 \%)$, but also in higher complete response rate $(14 \%$ vs. $0 \%)$, and OS rate (36\% vs. $7 \%$ ) [49].

Altogether, these trials included patients with different baseline characteristics, thus making it difficult to compare results. However, evidence to date has supported the role of anti-PD1 either as monotherapy or combined with ipilimumab, as a first-line therapeutic strategy for the treatment of patients with advanced unresectable/advanced mucosal melanoma. In this setting, multimodal treatment strategies combining radiotherapy with immunotherapy might lead to a boosted immune response with increased antitumor efficacy and improved local tumor control and symptoms relief [50-52].

Notably, clinical outcomes with ICIs seem to be poorer for Asian patients as compared with Caucasian patients with mucosal melanoma. In the KEYNOTE-151 trial, pembrolizumab provided an ORR of $13.3 \%$ in 15 Chinese patients with advanced/metastatic mucosal melanoma [53]. Similarly, the POLARIS-01 study showed poor clinical outcomes of toripalimab, a humanized antibody targeting PD1, among 22 Chinese patients with mucosal melanoma, with an ORR of $0 \%$, a median OS of 10.3 months, and a median PFS of 1.9 months [54]. Differences in the biologic profile, namely a higher prevalence of mutations affecting the WNT- $\beta$ catenin pathway and the high frequency of KIT mutations in an Asian population, might be responsible for the poor responses observed during treatment with ICIs [9,55-57]. 
Table 2. Overview of the main ongoing clinical trials (i.e., recruiting and active, not recruiting) for patients with mucosal melanoma in the adjuvant and metastatic setting (source: clinicaltrials.gov; and rctportal.niph.go.jp (accessed on 9 November 2021)).

\begin{tabular}{|c|c|c|c|c|c|}
\hline $\begin{array}{l}\text { Trial Name, } \\
\text { NCT Number }\end{array}$ & Type of Study & Condition (s) & Drug (s) & Estimated Sample Size & Primary Endpoint (s) \\
\hline \multicolumn{6}{|c|}{ Perioperative (including neoadjuvant and adjuvant) } \\
\hline NCT03178123 & Phase 2, randomized & $\begin{array}{c}\text { Mucosal melanoma that has been removed } \\
\text { by surgery }\end{array}$ & $\begin{array}{c}\text { toripalimab } \\
\text { high-dose recombinant IFN-a2b }\end{array}$ & $n=220$ & RFS (time frame: 5 years) \\
\hline NCT04180995 & Phase 2, single-arm & $\begin{array}{l}\text { Localized mucosal melanoma considered to } \\
\text { be able to be completely resected }\end{array}$ & $\begin{array}{l}\text { neoadjuvant toripalimab + axitinib (8 weeks); } \\
\text { adjuvant toripalimab (up to one year) }\end{array}$ & $n=30$ & $\begin{array}{l}\text { Pathological response rate } \\
\qquad(\mathrm{pCR} \text { and } \mathrm{pPR})\end{array}$ \\
\hline SALVO, NCT03241186 & Phase 2, single-arm & $\begin{array}{l}\text { Mucosal melanoma that has been removed } \\
\text { by surgery (R0 or R1) }\end{array}$ & $\begin{array}{l}\text { cycles 1-4: ipilimumab } 1 \mathrm{mg} / \mathrm{kg} \text { + nivolumab } \\
3 \mathrm{mg} / \mathrm{kg} \mathrm{q} 3 \mathrm{w} ; \\
\text { cycles 5-15: nivolumab } 480 \mathrm{mg} \text { q28d }\end{array}$ & $n=30$ & RFS \\
\hline $\begin{array}{c}\text { IMMUQ, } \\
\text { NCT03313206 }\end{array}$ & Phase 2, single-arm & $\begin{array}{l}\text { Resectable head and neck mucosal } \\
\text { melanoma, amenable of post-operative RT }\end{array}$ & $\begin{array}{l}\text { neoadjuvant pembrolizumab } 200 \mathrm{mg} \text { q3w (up to } 4 \text { doses); } \\
\text { adjuvant pembrolizumab } 200 \mathrm{mg} \text { q3w (up to one year) }\end{array}$ & $n=50$ & DFS \\
\hline NCT04622566 & Phase 2, single-arm & Resectable mucosal melanoma & $\begin{array}{l}\text { neoadjuvant pembrolizumab } 200 \mathrm{mg} \mathrm{q} 3 \mathrm{w} \text { + lenvatinib } \\
\text { QD (6 weeks); } \\
\text { adjuvant pembrolizumab } 200 \mathrm{mg} \text { q3w (up to one year) }\end{array}$ & $n=26$ & $\mathrm{pCR}$ rate \\
\hline NCT05111574 & Phase 2, randomized & $\begin{array}{l}\text { Mucosal melanoma that has been removed } \\
\text { by surgery }\end{array}$ & nivolumab + cabozantinib/placebo & $n=99$ & RFS \\
\hline MEL60, NCT02126579 & Phase $1 / 2$, randomized & Resected stage IIB/IV melanoma & LPV7 + TLR agonists & $n=62$ & $\begin{array}{l}\text { Incidence of AEs, T cell } \\
\text { response in peripheral blood }\end{array}$ \\
\hline NCT04879654 & Phase 2, single-arm & $\begin{array}{l}\text { Sinonasal melanoma removed with } \\
\text { endoscopic surgery }\end{array}$ & toripalimab $+\mathrm{CT}+\mathrm{RT}$ & $n=45$ & OS \\
\hline NCT02519322 & Phase 2, randomized & $\begin{array}{l}\text { Stage III or oligometastatic stage IV that can } \\
\text { be removed by surgery }\end{array}$ & $\begin{array}{l}\text { Arm A: neoadjuvant nivolumab q2w for } 4 \text { doses; } \\
\text { adjuvant nivolumab q2w for } 13 \text { doses } \\
\text { Arm B: neoadjuvant nivolumab + ipilimumab q3w for } \\
3 \text { doses; adjuvant nivolumab q2w for } 13 \text { doses } \\
\text { Arm C: neoadjuvant nivolumab + relatlimab q28d for } \\
2 \text { doses, adjuvant nivolumab + relatlimab q28d for } \\
10 \text { doses }\end{array}$ & $n=53$ & $\begin{array}{l}\text { Proportion of patients with } \\
\text { pathologic response to } \\
\text { neoadjuvant nivolumab and } \\
\text { ipilimumab plus } \\
\text { nivolumab therapy }\end{array}$ \\
\hline NCT03698019 & Phase 2, randomized & $\begin{array}{l}\text { Stage IIIB/C-IV resectable } \\
\text { high-risk melanoma }\end{array}$ & $\begin{array}{l}\text { Adjuvant pembrolizumab q3w for } 18 \text { cycles vs. } \\
\text { neoadjuvant pembrolizumab q3w for } 3 \text { cycles, followed } \\
\text { by adjuvant pembrolizumab q3w for } 15 \text { cycles }\end{array}$ & $n=500$ & EFS \\
\hline \multicolumn{6}{|c|}{ Metastatic } \\
\hline $\begin{array}{c}\text { MTAM, } \\
\text { NCT04472806 }\end{array}$ & Phase 2, single-arm & $\begin{array}{l}\text { Unresectable locally advanced/metastatic } \\
\text { mucosal melanoma }\end{array}$ & toripalimab + endostar $+\mathrm{CT}$ & $n=31$ & PFS \\
\hline NCT04318717 & Phase $1 / 2$, single-arm & Mucosal melanoma of the head and neck & pembrolizumab + hypofractionated RT & $n=16$ & Local tumor control rate \\
\hline
\end{tabular}


Table 2. Cont.

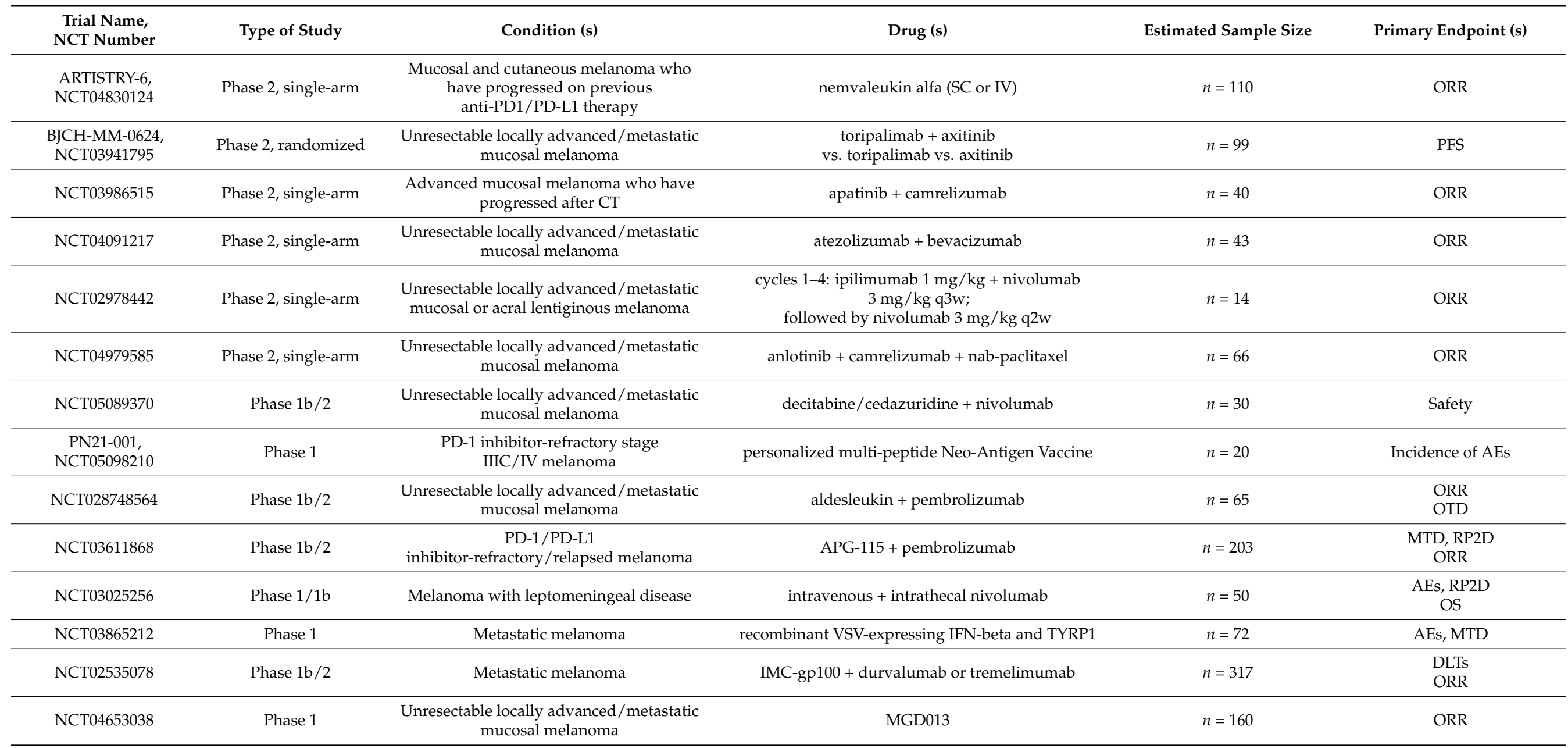

Abbreviations: AEs: adverse events; CT: chemotherapy; DFS: disease-free survival; DLT: dose-limiting toxicity; EFS: event-free survival; IFN: interferon; IV: intravenous; LPV7: long peptide vaccine 7; MTD: maximum tolerated dose; ORR: objective response rate; OS: overall survival; OTD: optimal tolerated dose; pCR: pathologic complete response; PFS: progressionfree survival; pPR: pathologic partial response; q28d: once every 28 days; q3w: once every three weeks; RP2D: recommended phase 2 dose; RT: radiotherapy; SC: subcutaneous; TLR: toll-like receptor; TYRP-1: tyrosinase related protein 1; VSV: Vesicular Stomatitis Virus. 


\section{Ongoing Clinical Trials and Future Perspectives}

Due to the limited efficacy of single-agent targeted and immunotherapy in mucosal melanoma, several combination strategies are currently under investigation in clinical trials (Table 2). Combination therapies aim at targeting multiple mechanisms by which the cancer cell proliferate and evade the immune surveillance, thus exerting a synergistic antitumor effect. Promising response rates and survival results have been reported with the combination of nivolumab and ipilimumab, as detailed in the previous section.

VEGF expression was associated with poor survival outcomes among patients with primary mucosal melanoma of the oral cavity, suggesting it could be a potential therapeutic target. Unfortunately, preliminary data of antiangiogenic drugs, either alone or combined with cytotoxic chemotherapy, have yielded only limited antitumor activity and therapeutic results $[37,58]$. Inhibition of the VEGF signaling pathway also exerts immune-mediated effects in the tumor microenvironment. The combination of VEGFR inhibitors and anti-PD1 antibodies has also been investigated among patients with mucosal melanoma, showing promising results. The randomized phase III trial LEAP-003 of pembrolizumab combined with lenvatinib for first-line treatment of metastatic melanoma also included patients with acral and mucosal melanoma and is currently ongoing (NCT03820986). Bevacizumab combined with the anti-PD-L1 antibody, atezolizumab, has been investigated in a phase II trial (NCT04091217). A phase 2 trial of the anti-PD1 antibody, camrelizumab in combination with the multitargeted tyrosine kinase inhibitor, anlotinib, and nab-paclitaxel as first-line treatment of mucosal melanoma is currently ongoing and recruiting patients (NCT04979585).

The combination of toripalimab and axitinib showed preliminary clinical activity in a population of 29 treatment-naïve Asian patients with metastatic mucosal melanoma in a phase IB clinical trial [59]. The ORR was 48.3\% (95\% CI, 29.4-67.5\%), and the median PFS was 7.5 months (95\% CI, 3.7-not reached). In this trial, PD-L1 expression and high tumor mutational burden (TMB) were associated with higher ORR and better PFS. Given the above-mentioned differences in prognosis and response to immunotherapy between Asian and non-Asian patients, this combination therapy should be validated in a randomized phase III trial, also including a non-Asian population, in order to confirm its activity.

Preliminary data from a phase II study showed promising activity of the combination of toripalimab with the multi-target tyrosine kinase inhibitor vorolanib (CM082). In this study ( $n=38$ patients evaluable for response), the ORR was $22.2 \%$, the disease control rate as 55.5\%, and median PFS 5.7 months (NCT03602547) [60].

Novel agents currently under evaluation in clinical trials for mucosal melanoma include the CDK inhibitor, dinaciblib (NCT00937937); the MDM2 inhibitor, APG-115, in combination with pembrolizumab (NCT03611868); the engineered interleukin 2, nemvaleukin alfa as a single agent (NCT04830124), and aldesleukin in combination with pembrolizumab (NCT02748564). Several trials are also evaluating the role of multimodal treatment integration (i.e., radiotherapy and radiosurgery) with immunotherapy in order to improve therapeutic results. Moreover, trials are underway in order to assess the efficacy of immunotherapy, either as single-agent anti-PD1 or as a combination of anti-PD1 and anti-CTLA4 as neoadjuvant and/or adjuvant treatments for locally advanced high-risk mucosal melanoma before and after surgery.

\section{Conclusions}

Mucosal melanoma is an aggressive disease with an overall poor prognosis. To date, only limited therapeutic benefits have been obtained with KIT inhibitors and immunotherapy (either as single-agent anti-PD1 or as a combination of anti-PD1 and anti-CTLA4). However, response rates and survival outcomes are still worse than those observed among patients with cutaneous melanoma. Increasing knowledge of the genetic profile of mucosal melanoma by means of whole genome and whole exome sequencing has allowed a better understanding of the biology of this disease and has paved the way for potential therapeutic targets. Further prospective studies are of utmost relevance in this setting, where standard therapies are less efficacious than in cutaneous melanoma. At the present time, several clini- 
cal trials are ongoing to assess the role of systemic treatment in the perioperative setting for potentially resectable disease and in the adjuvant setting after radical surgery for localized disease. Moreover, several combination strategies for advanced/metastatic or relapsed disease are under evaluation in clinical trials, mainly consisting in the combination of immunotherapy with radiotherapy or antiangiogenic drugs, but also with novel emerging compounds targeting other signaling pathways. Cooperative data collection in order to identify clinical factors that influence disease response to treatment will help us gain more insight and eventually improve outcomes of this rare disease.

Author Contributions: Conceptualization, A.I. and M.M.; methodology, A.I.; formal analysis, A.I. and D.M.; investigation, all authors; writing—original draft preparation, A.I. and M.M.; writing—review and editing, D.M., F.R. and F.G.; visualization, all authors; supervision, M.M. and F.R. All authors have read and agreed to the published version of the manuscript.

Funding: This research received no external funding.

Informed Consent Statement: Informed consent for publication was obtained from the patients or from their relatives in case of impossibility to obtain a signed consent form from the patient $\mathrm{him} /$ herself.

Data Availability Statement: No new data were created or analyzed in this study. Data sharing is not applicable to this article.

Conflicts of Interest: Alice Indini: Personal fees and honoraria for lectures: Brystol-Myers Squibb, MSD, Pierre Fabre, Sanofi, Novartis. Advisory Board for Novartis. Fausto Roila: None. Francesco Grossi: Honoraria for lectures and advisory board for: Novartis, Pfizer, Merck, Pierre Fabre, Boehringer Ingelheim. Honoraria for lectures: Amgen, AstraZeneca, Roche. Research funding, honoraria for lectures, and advisory board: Brystol-Myers Squibb. Daniela Massi: None. Mario Mandalà: None.

\section{References}

1. Scott, J.F.; Gerstenblith, M.R. Noncutaneous Melanoma. In Noncutaneous Melanoma; Codon Publications: Brisbane, Australia, 2018.

2. Dupin, E.; Le Douarin, N.M. Development of melanocyte precursors from the vertebrate neural crest. Oncogene 2003, 22, 3016-3023. [CrossRef] [PubMed]

3. MCLaughlin, C.C.; Wu, X.C.; Jemal, A.; Martin, H.J.; Roche, L.M.; Chen, V.W. Incidence of noncutaneous melanomas in the U.S. Cancer 2005, 103, 1000-1007. [CrossRef] [PubMed]

4. Chang, A.E.; Karnell, L.H.; Manck, H.R. The National Cancer Data Base Report on cutaneous and noncutaneous melanoma: A summary from 84,836 cases from the past decade. The American College of Surgeons Commission on Cancer and the American Cancer Society. Cancer 1998, 83, 1664-1678. [CrossRef]

5. Garbacz, T.; Osuchowski, M.; Bartosik-Psujek, H. Primary diffuse meningeal melanomatosis-A rare form of meningeal melanoma: Case report. BMC Neurol. 2019, 19, 271. [CrossRef]

6. Cameselle-Garcia, S.; Perez, J.L.F.; Areses, M.C.; de Castro, J.D.F.; Mosquera-Reboredo, J.; Garcia-Mata, J. Primary malignant melanoma of the biliary tract: A case report and literature review. World J. Clin. Cases 2019, 7, 2302-2308. [CrossRef]

7. Yang, C.; Sanchez-Vega, F.; Chang, J.C.; Chatila, W.K.; Shoushtari, A.; Ladanyi, M.; Travis, W.D.; Busam, K.J.; Rekhtman, N. Lung-only melanoma: UV mutational signature supports origin from occult cutaneous primaries and argues against the concept of primary pulmonary melanoma. Mod. Pathol. 2020, 33, 2244-2255. [CrossRef]

8. Van Zeijl, M.C.T.; Boer, F.L.; van Poelgeest, M.I.E.; van den Eertwegh, A.J.M.; Wouters, M.W.J.M.; de Wreede, L.C.; Aarts, M.J.B.; van den Berkmortel, F.W.P.J.; de Groot, J.W.B.; Hospers, G.A.P.; et al. Survival outcomes of patients with advanced mucosal melanoma diagnosed from 2013 to 2017 in the Netherlands-A nationwide population-based study. Eur. J. Cancer 2020, 137, 127-135. [CrossRef] [PubMed]

9. Nassar, K.W.; Tan, A.C. The mutational landscape of mucosal melanoma. Semin. Cancer Biol. 2020, 61, 139-148. [CrossRef]

10. Newell, F.; Kong, Y.; Wilmott, J.S.; Johansson, P.A.; Ferguson, P.M.; Cui, C.; Li, Z.; Kazakoff, S.H.; Burke, H.; Dodds, T.J.; et al. Whole-genome landscape of mucosal melanoma reveals diverse drivers and therapeutic targets. Nat. Commun. 2019, 10, 3163. [CrossRef]

11. Zhou, R.; Shi, C.; Tao, W.; Li, J.; Wu, J.; Han, Y.; Yang, G.; Gu, Z.; Xu, S.; Wang, Y.; et al. Analysis of mucosal melanoma whole-genome landscapes reveals clinically relevant genomic aberrations. Clin. Cancer Res. 2019, 25, 3548-3560. [CrossRef]

12. Iida, Y.; Salomon, M.P.; Hata, K.; Tran, K.; Ohe, S.; Griffiths, C.F.; Hsu, S.C.; Nelson, N.; Hoon, D.S.B. Predominance of triple wild-type and IGF2R mutations in mucosal melanomas. BMC Cancer 2018, 18, 1054. [CrossRef]

13. Zebary, A.; Jangard, M.; Omholt, K.; Ragnarsson-Olding, B.; Hansson, J. KIT, NRAS and BRAF in sinonasal mucosal melanoma: A study of 56 cases. Br. J. Cancer 2013, 109, 559-564. [CrossRef] [PubMed] 
14. Amit, M.; Tam, S.; Abdelmeguid, A.S.; Roberts, D.B.; Takahashi, Y.; Raza, S.M.; Su, S.Y.; Kupferman, M.E.; DeMonte, F.; Hanna, E.Y. Mutation status among patients with sinonasal mucosal melanoma and its impact on survival. Br. J. Cancer 2017, 116, 1564-1571. [CrossRef]

15. Ozturk Sari, S.; Yilmaz, I.; Taskin, O.C.; Narli, G.; Sen, F.; Comoglu, S.; Firat, P.; Bilgic, B.; Yilmazbayhan, D.; Ozluk, Y.; et al. BRAF, NRAS, KIT, TERT, GNAQ/GNA11 mutation profile analysis of head and neck mucosal melanomas: A study of 42 cases. Pathology 2017, 49, 55-61. [CrossRef] [PubMed]

16. Hou, J.Y.; Baptiste, C.; Hombalegowda, R.B.; Tergas, A.I.; Feldamn, R.; Jones, N.L.; Chatterjee-Paer, R.; Bus-Kwolfski, A.; Wright, J.D.; Burke, W.M. Vulvar and vaginal melanoma: A unique subclass of mucosal melanoma based on a comprehensive molecular analysis of 51 cases compared with 2253 cases of nongynecologic melanoma. Cancer 2017, 123, 1333-1344. [CrossRef]

17. Ablain, J.; Xu, M.; Rothschild, H.; Jordan, R.C.; Mito, J.K.; Daniels, B.H.; Bell, C.F.; Joseph, N.M.; Wu, H.; Bastian, B.C.; et al. Human tumor genomics and zebrafish modeling identify SPRED1 loss as a driver of mucosal melanoma. Science 2018, 362, 1055-1060. [CrossRef] [PubMed]

18. Dumaz, N.; Jouenne, F.; Delyon, J.; Mourah, S.; Bensussan, A.; Lebbé, C. Atypical BRAF and NRAS Mutations in Mucosal Melanoma. Cancers 2019, 11, 1133. [CrossRef]

19. Kim, H.S.; Jung, M.; Kang, H.N.; Kim, H.; Park, C.W.; Kim, S.M.; Shin, S.J.; Kim, S.H.; Kim, S.G.; Kim, E.K.; et al. Oncogenic BRAF fusions in mucosal melanomas activate the MAPK pathway and are sensitive to MEK/PI3K inhibition or MEK/CDK4/6 inhibition. Oncogene 2017, 36, 3334-3345. [CrossRef]

20. Wróblewska, J.P.; Dias-Santagata, D.; Ustaszewski, A.; Wu, C.L.; Fujimoto, M.; Selim, M.A.; Biernat, W.; Ryś, J.; Marszalek, A.; Hoang, M.P. Prognostic Roles of BRAF, KIT, NRAS, IGF2R and SF3B1 Mutations in Mucosal Melanomas. Cells 2021, $10,2216$. [CrossRef]

21. Hodis, E.; Watson, I.R.; Kryukov, G.V.; Arold, S.T.; Imielinski, M.; Theurillat, J.P.; Nickerson, E.; Auclair, D.; Li, L.; Place, C.; et al. A landscape of driver mutations in melanoma. Cell 2012, 150, 251-263. [CrossRef]

22. Hintzsche, J.D.; Gorden, N.T.; Amato, C.M.; Kim, J.; Wuensch, K.E.; Robinson, S.E.; Applegate, A.J.; Couts, K.L.; Medina, T.M.; Wells, K.R.; et al. Whole exome sequencing identifies recurrent SF3B1 R625 mutation and comutation of NF1 and KIT in mucosal melanoma. Melanoma Res. 2017, 27, 189-199. [CrossRef] [PubMed]

23. Wang, L.; Brooks, A.N.; Fan, J.; Wan, Y.; Gambe, R.; Li, S.; Hergert, S.; Yin, S.; Freeman, S.S.; Levin, J.Z.; et al. Transcriptomic Characterization of SF3B1 Mutation Reveals Its Pleiotropic Effects in Chronic Lymphocytic Leukemia. Cancer Cell. 2016, 30, 750-763. [CrossRef] [PubMed]

24. Yang, H.M.; Hsiao, S.J.; Schaeffer, D.F.; Lai, C.; Remotti, H.E.; Horst, D.; Mansukhani, M.M.; Horst, B.A. Identification of recurrent mutational events in anorectal melanoma. Mod. Pathol. 2017, 30, 286-296. [CrossRef] [PubMed]

25. Yavuzyigitoglu, S.; Koopmans, A.E.; Verdijk, R.M.; Vaarwater, J.; Eussen, B.; van Bodegom, A.; Paridaens, D.; Kilic, E.; de Klein, A.; Rotterdam Ocular Melanoma Study Group. Uveal melanomas with SF3B1 mutations: A distinct subclass associated with late onset metastases. Ophthalmology 2016, 123, 1118-1128. [CrossRef] [PubMed]

26. Couts, K.L.; Bemis, J.; Turner, J.A.; Bagby, S.M.; Murphy, D.; Christiansen, J.; Hintzsche, J.D.; Le, A.; Pitts, T.M.; Wells, K.; et al. ALK inhibitor response in melanomas expressing EML4-ALK fusions and alternate ALK isoforms. Mol. Cancer Ther. 2018, 17, 222-231. [CrossRef]

27. Kalbasi, A.; Ribas, A. Tumour-intrinsic resistance to immune-checkpoint blockade. Nat. Rev. Immunol. 2020, 20, 25-39. [CrossRef]

28. Yu, J.; Yan, J.; Guo, Q.; Chi, Z.; Tang, B.; Zheng, B.; Yu, J.; Yin, T.; Cheng, Z.; Wu, X.; et al. Genetic Aberrations in the CDK4 Pathway Are Associated with Innate Resistance to PD-1 Blockade in Chinese Patients with Non-Cutaneous Melanoma. Clin. Cancer Res. 2019, 25, 6511-6523. [CrossRef]

29. Davoli, T.; Uno, H.; Wooten, E.C.; Elledge, S.J. Tumor aneuploidy correlates with markers of immune evasion and with reduced response to immunotherapy. Science 2017, 255, 6322. [CrossRef]

30. Jurmeister, P.; Wrede, N.; Hoffmann, I.; Vollbrecht, C.; Heim, D.; Hummel, M.; Wolkenstein, P.; Koch, I.; Heynol, V.; Schmitt, W.D.; et al. Mucosal melanomas of different anatomic sites share a common global DNA methylation profile with cutaneous melanoma but show location-dependent patterns of genetic and epigenetic alterations. J. Pathol. 2021. Epub ahead of print. [CrossRef]

31. Cirenajwis, H.; Lauss, M.; Ekedahl, H.; Törngren, T.; Kvist, A.; Saal, L.H.; Olsson, H.; Staaf, J.; Carneiro, A.; Ingvar, C.; et al. NF1-mutated melanoma tumors harbor distinct clinical and biological characteristics. Mol. Oncol. 2017, 11, 438-451. [CrossRef]

32. Carvajal, R.D.; Antonescu, C.R.; Wolchok, J.D.; Chapman, P.B.; Roman, R.A.; Teitcher, J.; Panageas, K.S.; Busam, K.J.; Chmielowski, B.; Lutzky, J.; et al. KIT as a therapeutic target in metastatic melanoma. JAMA 2011, 305, 2327-2334. [CrossRef] [PubMed]

33. Hodi, F.S.; Corless, C.L.; Giobbie-Hurder, A.; Fletcher, J.A.; Zhu, M.; Marino-Enriquez, A.; Friedlander, P.; Gonzalez, R.; Weber, J.S.; Gajewski, T.F.; et al. Imatinib for melanomas harboring mutationally activated or amplified KIT arising on mucosal, acral, and chronically sun-damaged skin. J. Clin. Oncol. 2013, 31, 3182-3190. [CrossRef]

34. Steeb, T.; Wessely, A.; Petzold, A.; Kohl, C.; Erdmann, M.; Berking, C.; Heppt, M.V. c-Kit inhibitors for unresectable or metastatic mucosal, acral or chronically sun-damaged melanoma: A systematic review and one-arm meta-analysis. Eur. J. Cancer 2021, 157, 348-357. [CrossRef]

35. Delyon, J.; Chevret, S.; Jouary, T.; Dalac, S.; Dalle, S.; Guillot, B.; Arnault, J.P.; Avril, M.F.; Bedane, C.; Bens, G.; et al. STAT3 Mediates Nilotinib Response in KIT-Altered Melanoma: A Phase II Multicenter Trial of the French Skin Cancer Network. J. Investig. Dermatol. 2018, 138, 58-67. [CrossRef] [PubMed] 
36. Tang, B.; Sheng, X.; Kong, Y.; Chi, Z.; Si, L.; Cui, C.; Yan, X.; Mao, L.; Lian, B.; Li, S.; et al. Palbociclib for treatment of metastatic melanoma with copy number variations of CDK4 pathway: Case report. Chin. Clin. Oncol. 2018, 7, 62. [CrossRef] [PubMed]

37. Mao, L.; Dai, J.; Cao, Y.; Bai, X.; Sheng, X.; Chi, Z.; Cui, C.; Kong, Y.; Zhang, Y.; Wu, L.; et al. Palbociclib in advanced acral melanoma with genetic aberrations in the cyclin-dependent kinase 4 pathway. Eur. J. Cancer 2021, 148, 297-306. [CrossRef]

38. Steensma, D.P.; Wermke, M.; Klimek, V.M.; Greenberg, P.L.; Font, P.; Komrokji, R.S.; Yang, J.; Brunner, A.M.; Carraway, H.E.; Ades, L.; et al. Phase I First-in-Human Dose Escalation Study of the oral SF3B1 modulator H3B-8800 in myeloid neoplasms. Leukemia 2021. Epub ahead of print. [CrossRef]

39. Cui, C.; Zhou, L.; Lian, B.; Si, L.; Sheng, X.; Chi, Z.; Kong, Y.; Wang, X.; Tang, B.; Mao, L.; et al. Safety and Efficacy of Apatinib Combined with Temozolomide in Advanced Melanoma Patients after Conventional Treatment Failure. Transl. Oncol. 2018, 11, 1155-1159. [CrossRef]

40. Moya-Plana, A.; Herrera Gómez, R.G.; Rossoni, C.; Dercle, L.; Ammari, S.; Girault, I.; Roy, S.; Scoazec, J.Y.; Vagner, S.; Janot, F.; et al. Evaluation of the efficacy of immunotherapy for non-resectable mucosal melanoma. Cancer Immunol. Immunother. 2019, 68, 1171-1178. [CrossRef]

41. Mignard, C.; Deschamps Huvier, A.; Gillibert, A.; Duval Modeste, A.B.; Dutriaux, C.; Khammari, A.; Avril, M.F.; Kramkimel, N.; Mortier, L.; Marcant, P.; et al. Efficacy of Immunotherapy in Patients with Metastatic Mucosal or Uveal Melanoma. J. Oncol. 2018, 2018, 1908065. [CrossRef]

42. Shoushtari, A.N.; Munhoz, R.R.; Kuk, D.; Ott, P.A.; Johnson, D.B.; Tsai, K.K.; Rapisuwon, S.; Eroglu, Z.; Sullivan, R.J.; Luke, J.J.; et al. The efficacy of anti-PD-1 agents in acral and mucosal melanoma. Cancer 2016, 122, 3354-3362. [CrossRef] [PubMed]

43. Postow, M.A.; Luke, J.J.; Bluth, M.J.; Ramaiya, N.; Panageas, K.S.; Lawrence, D.P.; Ibrahim, N.; Flaherty, K.T.; Sullivan, R.J.; Ott, P.A.; et al. Ipilimumab for patients with advanced mucosal melanoma. Oncologist 2013, 18, 726-732. [CrossRef]

44. Hamid, O.; Robert, C.; Ribas, A.; Hodi, F.S.; Walpole, E.; Daud, A.; Arance, A.S.; Brown, E.; Hoeller, C.; Mortier, L.; et al. Antitumour activity of pembrolizumab in advanced mucosal melanoma: A post-hoc analysis of KEYNOTE-001, 002, 006. Br. J. Cancer 2018, 119, 670-674. [CrossRef] [PubMed]

45. Nathan, P.; Ascierto, P.A.; Haanen, J.; Espinosa, E.; Demidov, L.; Garbe, C.; Guida, M.; Lorigan, P.; Chiarion-Sileni, V.; Gogas, H.; et al. Safety and efficacy of nivolumab in patients with rare melanoma subtypes who progressed on or after ipilimumab treatment: A single-arm, open-label, phase II study (CheckMate 172). Eur. J. Cancer 2019, 119, 168-178. [CrossRef]

46. D'Angelo, S.P.; Larkin, J.; Sosman, J.A.; Lebbé, C.; Brady, B.; Neyns, B.; Schmidt, H.; Hassel, J.C.; Hodi, F.S.; Lorigan, P.; et al Efficacy and Safety of Nivolumab Alone or in Combination with Ipilimumab in Patients With Mucosal Melanoma: A Pooled Analysis. J. Clin. Oncol. 2017, 35, 226-235. [CrossRef]

47. Rose, A.A.N.; Armstrong, S.M.; Hogg, D.; Butler, M.O.; Saibil, S.D.; Arteaga, D.P.; Pimentel Muniz, T.; Kelly, D.; Ghazarian, D.; King, I.; et al. Biologic subtypes of melanoma predict survival benefit of combination anti-PD1+anti-CTLA4 immune checkpoint inhibitors versus anti-PD1 monotherapy. J. Immunother. Cancer 2021, 9, e001642. [CrossRef] [PubMed]

48. Klemen, N.D.; Wang, M.; Rubinstein, J.C.; Olino, K.; Clune, J.; Ariyan, S.; Cha, C.; Weiss, S.A.; Kluger, H.M.; Sznol, M. Survival after checkpoint inhibitors for metastatic acral, mucosal and uveal melanoma. J Immunother Cancer 2020, 8, e000341. [CrossRef] [PubMed]

49. Shoushtari, A.N.; Wagstaff, J.; Ascierto, P.A.; Butler, M.O.; Lao, C.D.; Marquez-Rodas, I.; Chiarion-Sileni, V.; Dummer, R.; Ferrucci, P.F.; Lorigan, P.; et al. CheckMate 067: Long-term outcomes in mucosal melanoma. J. Clin. Oncol. 2020, 38 , 10019. [CrossRef]

50. Teterycz, P.; Czarnecka, A.M.; Indini, A.; Spałek, M.J.; Labianca, A.; Rogala, P.; Cybulska-Stopa, B.; Quaglino, P.; Ricardi, U.; Badellino, S.; et al. Multimodal Treatment of Advanced Mucosal Melanoma in the Era of Modern Immunotherapy. Cancers 2020, 12, 3131. [CrossRef]

51. Umeda, Y.; Yoshikawa, S.; Kiniwa, Y.; Maekawa, T.; Yamasaki, O.; Isei, T.; Matsushita, S.; Nomura, M.; Nakai, Y.; Fukushima, S.; et al. Real-world efficacy of anti-PD-1 antibody or combined anti-PD-1 plus anti-CTLA-4 antibodies, with or without radiotherapy, in advanced mucosal melanoma patients: A retrospective, multicenter study. Eur. J. Cancer 2021, 157, 361-372. [CrossRef]

52. Kim, H.J.; Chang, J.S.; Roh, M.R.; Oh, B.H.; Chung, K.Y.; Shin, S.J.; Koom, W.S. Effect of Radiotherapy Combined With Pembrolizumab on Local Tumor Control in Mucosal Melanoma Patients. Front. Oncol. 2019, 9, 835. [CrossRef]

53. Si, L.; Zhang, X.; Shu, Y.; Pan, H.; Wu, D.; Liu, J.; Lou, F.; Mao, L.; Wang, X.; Wen, X.; et al. A Phase Ib Study of Pembrolizumab as Second-Line Therapy for Chinese Patients With Advanced or Metastatic Melanoma (Keynote-151). Transl. Oncol. 2019, 12, 828-835. [CrossRef] [PubMed]

54. Tang, B.; Chi, Z.; Chen, Y.; Liu, X.; Wu, D.; Chen, J.; Song, X.; Wang, W.; Dong, L.; Song, H.; et al. Safety, Efficacy, and Biomarker Analysis of Toripalimab in Previously Treated Advanced Melanoma: Results of the POLARIS-01 Multicenter Phase II Trial. Clin. Cancer Res. 2020, 26, 4250-4259. [CrossRef] [PubMed]

55. Sakaizawa, K.; Ashida, A.; Uchiyama, A.; Ito, T.; Fujisawa, Y.; Ogata, D.; Matsushita, S.; Fujii, K.; Fukushima, S.; Shibayama, Y.; et al. Clinical characteristics associated with BRAF, NRAS and KIT mutations in Japanese melanoma patients. J. Dermatol. Sci. 2015, 80, 33-37. [CrossRef]

56. Nakamura, Y.; Namikawa, K.; Yoshikawa, S.; Kiniwa, Y.; Maekawa, T.; Yamasaki, O.; Isei, T.; Matsushita, S.; Nomura, M.; Nakai, Y.; et al. Anti-PD-1 antibody monotherapy versus anti-PD-1 plus anti-CTLA-4 combination therapy as first-line immunotherapy in unresectable or metastatic mucosal melanoma: A retrospective, multicenter study of 329 Japanese cases (JMAC study). ESMO Open 2021, in press. [CrossRef] 
57. Bai, X.; Shoushtari, A.N.; Warner, A.B.; Quach, H.; Cann, C.G.; Zhang, M.; Pallan, L.; Harvey, C.; Si, L.; Tang, B.; et al. Discrepancies in response and immune-related adverse events (irAEs) of anti-PD-1 monotherapy between races and primary sites in patients (pts) with advanced nonacral cutaneous melanoma (NACM). J. Clin. Oncol. 2021, 39 (Suppl. S15), 9530. [CrossRef]

58. Kim, K.B.; Sosman, J.A.; Fruehauf, J.P.; Linette, G.P.; Markovic, S.N.; McDermott, D.F.; Weber, J.S.; Nguyen, H.; Cheverton, P.; Chen, D.; et al. BEAM: A randomized phase II study evaluating the activity of bevacizumab in combination with carboplatin plus paclitaxel in patients with previously untreated advanced melanoma. J. Clin. Oncol. 2012, 30, 34-41. [CrossRef]

59. Sheng, X.; Yan, X.; Chi, Z.; Si, L.; Cui, C.; Tang, B.; Li, S.; Mao, L.; Lian, B.; Wang, X.; et al. Axitinib in Combination with Toripalimab, a Humanized Immunoglobulin G4 Monoclonal Antibody Against Programmed Cell Death-1, in Patients with Metastatic Mucosal Melanoma: An Open-Label Phase IB Trial. J. Clin. Oncol. 2019, 37, 2987-2999. [CrossRef]

60. Si, L.; Sheng, X.; Mao, L.; Li, C.; Wang, X.; Bai, X.; Qi, Z.H.; Chi, Z.; Cui, C.; Lian, B.; et al. A phase II study of vorolanib (CM082) in combination with toripalimab (JS001) in patients with advanced mucosal melanoma. J. Clin. Oncol. 2020, 38 (Suppl. S15), 10040. [CrossRef] 